\title{
SKYMONITOR: A Global Network for Night Sky Brightness Measurements
}

\author{
Dan McKenna, ${ }^{1}$ Donald Davis,${ }^{2}$ and Paul Boley ${ }^{2}$ \\ ${ }^{1}$ 1Palomar Observatory Po Box 200, Palomar Mountain CA 92060 \\ email: dmck@astro.caltech.edu \\ ${ }^{2}$ International Dark-Sky Association, 3223 N. First Ave., Tucson, Arizona 85719 \\ email: drd@psi.edu and pboley@gmail.com
}

\begin{abstract}
We have deployed a network of autonomous photometers that continuously measures the night sky brightness in the visual region at two sky positions simultaneously, typically near the zenith and the second at an elevation angle of 20 degrees. The Photometers are calibrated as a network to better than 5 .
\end{abstract}

Keywords. night sky brightness, networked photometers, SKYMONITOR

\section{Overview}

The SKYMONITOR network uses two, 4 degree field of view, silicon photo detectors filtered by Hoya CM500 glass to cover a wavelength range of $320 \mathrm{~nm}$ to $750 \mathrm{~nm}$. The brightness data are temperature corrected and calibrated before leaving the manufacturing facility for an estimated variation in calibration of less than $3 \%$. Comparing several photometers co-located and pointed, we find that the variation between photometers of the observed sky brightness is primarily due to differences in the field of view. We conclude that our photometers need to be pointed better than 1/10 the field of view especially when dealing with areas of the sky with high gradients such as near the galactic plane or near the horizon.

\section{Current Results}

Photometers as tested in the field by on sky inter comparison vary by less than $5 \%$. Variations in field of view are mainly due to detector packaging and alignment between optical and mechanical axis and are the largest components of uncertainty. The current preliminary photometric zero point is about +0.52 Magnitudes different from a calibration based on Vega. Our estimated sky brightness for the darkest skies is currently 21.84 corrected to $\mathrm{V}$ magnitude. Long term stability will be monitored by periodically retesting photometer sensors in the lab, the observation of bright stars and the galactic plane. The current generation of Photometers transmits raw brightness measurements and sensor temperature data every 30 minutes to the IDA collection center in Tucson where the calibration is applied. The data may be viewed on line at nsbm.darksky.org. 